\title{
Vers une conceptualisation de la notion de « français régional » : de la dialectologie à la sociolinguistique
}

Towards a conceptualization of the notion of regional French: from dialectology to sociolinguistics

Hacia una conceptualización de la noción de "francés regional»: desde la dialectología a la sociolingüística

Marie-Madeleine Bertucci

\section{CpenEdition}

\section{Journals}

Édition électronique

URL : https://journals.openedition.org/mots/25404

DOI : $10.4000 /$ mots. 25404

ISSN : 1960-6001

Éditeur

ENS Éditions

Édition imprimée

Date de publication : 11 juillet 2019

Pagination : 167-183

ISBN : 979-10-362-0170-7

ISSN : 0243-6450

Référence électronique

Marie-Madeleine Bertucci, « Vers une conceptualisation de la notion de « français régional » : de la dialectologie à la sociolinguistique », Mots. Les langages du politique [En ligne], 120 | 2019, mis en ligne le 01 janvier 2022, consulté le 23 avril 2022. URL : http://journals.openedition.org/mots/25404 ; DOI : https://doi.org/10.4000/mots.25404 


\section{Vers une conceptualisation de la notion de « français régional " : de la dialectologie à la sociolinguistique}

La période qui s’étend de la fin du XIXe siècle aux années 1950 est marquée par la diffusion de la variété de français qu'on désigne ordinairement comme le «français de Paris », identifiée comme le français des locuteurs «cultivés» et ressentie d'un point de vue normatif comme le français standard (Francard, 2010, p. 113), autrement dit «l'usage dominant par rapport aux variations effectives que présente toute langue» (Riegel, Pellat, Rioul, 2016, p.19).

Une telle perception, qui hiérarchise les variétés de langues en présence ainsi que leurs locuteurs, suggère les tensions qui sous-tendent les relations entre ces variétés et le caractère potentiellement conflictuel de leurs confrontations. En effet, la visée des représentations forgées à propos des variétés en présence est homogénéisante. Elle procède d'une conception monolingue, fondée sur des raisons politiques, et contribue au renforcement de l'unité nationale, en imposant la variété retenue ${ }^{1}$ - parmi d'autres - pour être la variété de référence. Cette conception procède

[d’]une vision centralisatrice et idéalisée de la langue, loin de toute variation diatopique ou diastratique, à la fois dans le travail de grammatisation et dans l'enseignement de la langue. (Francard, 2010, p.113)²

Le rôle de l'institution scolaire a été déterminant pour atténuer, voire dissimuler les processus variationnels et accroître la visibilité de la variété de référence, du fait des enjeux politiques des contenus de l'enseignement du français dans les programmes scolaires, lesquels ont explicitement pour fonction de contribuer à «fortifier l'unité nationale », ainsi que l'indique l'extrait suivant

1. Le «français de Paris » confondu avec le «français national » par exemple.

2. La variation diatopique est également dénommée spatiale, géographique, régionale, locale. La variation diastratique est également dénommée sociale (Gadet, 2007 [2003], p. 23). 
des Programmes et instructions commentés pour l'enseignement du premier degré3, en 1923 :

Le maître doit se proposer pour but d'amener ces enfants à exprimer leurs pensées et leurs sentiments en un langage correct. [...] Nos instituteurs affronteront, pour le remplir, tous les obstacles, car ils sentent bien que donner l'enseignement du français, ce n'est pas seulement travailler au maintien et à l'expansion d'une belle langue et d'une belle littérature, c'est fortifier l'unité nationale. (Bérard, 1995 [1923], p.324)

En second lieu, l'école a pour mission d'enseigner la variété standard de manière à homogénéiser les pratiques langagières. Les Instructions de 1938 pour l'enseignement du premier degré opposent explicitement la langue parlée par les élèves, qu’ils stigmatisent, à celle enseignée par les maîtres, laquelle est présentée comme «l'usage correct, le bon usage»:

La langue française que les maîtres enseignent n'est pas celle que les enfants emploient spontanément. Les enfants ont acquis en parlant [...] les habitudes linguistiques de leur milieu. Ils parlent une langue mêlée de mots d'argot et de termes impropres. [...] À l'école, les maîtres enseignent l'usage correct, le bon usage de la langue [...]. (Zay, 1938, p.14)

Le contexte général de cette première moitié du xxe siècle est également propice à l'imposition d'une variété. Les transformations sociales qui suivirent la Première Guerre mondiale et surtout le déclin de la société rurale, marquée par l'exode des agriculteurs vers les villes (Rémond, 1974, p. 45), se caractérisent par des modifications de la situation linguistique et par la montée en puissance du français au détriment des formes diatopiques (Dauzat, 1933, p.135).

Dans le même temps, on voit émerger dans les préoccupations des linguistes un intérêt pour des formes qu'ils n'attribuent ni aux «patois» ni au français standard, qui sont ancrées dans une réalité géographique et qu'ils interprètent comme la manifestation d'une variation diatopique, fortement liée aux "patois», mais qui est néanmoins perçue comme étant du français. Cette variété est évoquée de manière stigmatisante et condescendante. Sa perception s'inscrit dès le départ dans une «perspective globalement négative», celle de «l'idéologie glottophobe hégémonique» dont parle Philippe Blanchet (2016, p. 19).

Ces formes sont progressivement perçues comme relevant des français présents dans différentes zones géographiques (Taverdet, Straka, 1977) et désignées comme des «français régionaux», et non plus comme des «patois » ou des «dialectes». En effet, la description géographique, qui atomise les pratiques langagières en une «multitude de parlers » (Baggioni, 1993, p. 26), paraît montrer ses limites. Les notions de «patois» et de «dialectes» ne semblent

3. Pour le Cours préparatoire, élémentaire et moyen. 
plus adaptées pour rendre compte de «l'hétérogénéité linguistique » et du «principe plus transversal et processuel de variation » qui caractérisent ces formes (Blanchet, 2016, p. 22). Compte tenu des limites du cadre théorique communément admis jusque-là, la dénomination de «français régional» émerge peu à peu du fait d'une plus grande sensibilité à la dimension diastratique et à la montée en puissance de la notion de norme endogène, qui impliquent une prise de distance avec les notions de «patois» et de «dialectes». Outre leurs spécificités linguistiques, les français régionaux constituent pour les locuteurs « un composant identitaire important» (Bertucci, 2016, p.12), lequel minimise l'importance du seul critère diatopique. Le palier de réflexion que constitue la région gagnerait également à être davantage traité dans sa dimension politique qu'il ne l'est. Dans un contexte mondialisé, la dimension régionale, ou régionalisation4, peut en effet apparaître comme particulièrement adaptée à une réflexion sur le français régional en raison de sa fonction d'échelle de régulation5.

L'étude se développera en trois temps. On verra d'abord comment la notion de français régional a évolué des premiers travaux d’Albert Dauzat jusqu'aux analyses contemporaines. Ensuite, on étudiera l'émergence de la notion de norme endogène et les apports des études sociolinguistiques consacrées aux français non hexagonaux et notamment ceux de la lexicographie francophone. Enfin, on s'interrogera sur la modernité de la notion de région dans le contexte mondialisé contemporain et sur la revitalisation de la notion de français régional qu'elle permet.

\section{Évolution de la notion de français régional}

\section{La variation géographique}

Si l'on essaie de retracer les grandes lignes de l'évolution de la notion de français régional (désormais FR), on s'aperçoit que, depuis les premiers travaux d'A. Dauzat (1906), c'est la dimension de variation géographique, ou diatopique, ou topolectale, du français, observable en synchronie (Pöll, 2001, p. 25), qu'on retrouve le plus fréquemment dans les recherches, qui pensent les FR en termes de continuum géolinguistique (Baggioni, 1993, p. 26). À partir des années 1970, les linguistes s'intéressèrent particulièrement à l'étude des variétés topolectales du français, dans l'espace francophone, comme l'illustrent les exemples des recherches effectuées au Québec (Poirier, 1995) ou en Wallonie (Francard, 1993).

4. On parlera de régionalisation et non de régionalisme, pour éviter toute confusion.

5. L’intégration régionale est une notion économique. On la définit comme une des échelles de régulation de l'économie mondiale (Hugon éd., 2002, p. 9). Les liens des langues régionales avec le développement économique ont d'ailleurs déjà été étudiés (Huck, Kahn, 2009). 
On peut donc se demander ce qu'on entend sous le terme de FR. S'il est clair qu'on admet généralement que le FR n'a rien de commun avec les dialectes ou des langues nettement identifiées comme telles (basque, occitan, etc.), on reconnaît également qu'il existe néanmoins des différences sensibles entre les français.

\section{La rupture avec la tradition dialectologique}

Dans une perspective dialectologique plus étroite, on a décrit le FR comme «l'ensemble des variantes géolinguistiques du français» (Tuaillon, 1977, p. 8 ; Taverdet, 1977, p. 41). Certains chercheurs considèrent même que le FR n'existe pas (Tuaillon, 1983, p. 19).

Daniel Baggioni attribue ces réticences à l'égard du FR, et en particulier celles de Gaston Tuaillon, à un conflit de type disciplinaire né dans les années 1980, lequel viendrait des difficultés de la dialectologie, discipline implantée de longue date et ayant produit des travaux reconnus, à prendre un tournant sociolinguistique, qui la conduirait à admettre l'affaiblissement des réalités dialectales, à analyser les faits de langue avec une orientation scientifique différente, et à remettre en question des méthodologies et un cadre théorique un peu vieillissants (Baggioni, 1995, p. 68-69). La notion de FR a constitué pour la dialectologie un véritable obstacle épistémologique pour trois raisons. D’abord, la notion est en rupture avec la tradition héritée de la linguistique historique et revendiquée par la dialectologie, qui vise à décrire les faits linguistiques en termes de survivances, de traces d'une évolution cherchant à rendre compte du changement linguistique (ibid., p. 68). Ensuite, plus synthétique, elle s'oppose aux analyses fondant la description dialectologique de la variation sur le modèle d'un continuum géolinguistique, qui atomise les faits de langue en une multitude de parlers (loc. cit.), là où une analyse sociolinguistique opérerait des regroupements en des ensembles plus vastes, en fonction de critères le plus souvent sociopolitiques. Enfin, l'analyse des dialectes en tant que systèmes autonomes n'a pas conduit la dialectologie à s’intéresser à des variétés de français venant s'intercaler entre le français de référence et les dialectes, ces derniers étant apparentés ou non à la variété de référence. La dialectologie n'a pas intégré non plus, dans ses analyses, la question des situations de contacts de langues (Baggioni, 1993, p. 25). Ainsi, les FR ont pu être identifiés à ce moment-là, tant par la dialectologie que par la sociolinguistique, comme un stade intermédiaire, interne au changement de langue et envisagé comme une survivance substratique (Tuaillon, 1983, p.19; Walter, 1984, p. 190 ; Baggioni, 1993, p. 26). 
Un échelon intermédiaire entre la langue régionale et le français commun

Bodo Müller, dans les années 1980, parlait du FR comme d'un niveau régional intermédiaire entre la langue régionale et le français commun (Müller, 1985, p.158). Cette notion d'échelon intermédiaire permet d'aborder la question de la hiérarchie présente dans certaines descriptions, le FR y étant subordonné au français standard, qui incarne la norme de référence. D. Baggioni, dans un article intitulé «Historique du concept de français régional», soulignait la fonction sociolinguistique du $\mathrm{FR}$ et sa place entre le français de référence et les "dialectes/patois». La notion de FR «vient remplir un creux entre le français, réalité d'évidence non problématisée [...] et le dialecte/patois, réalité indigne de considération » (Baggioni, 1993, p. 17). La sensibilité à des pratiques distinctes s'est d'abord traduite par une réaction normative qui tendait à sanctionner comme faute tout usage senti comme déviant alors qu'il pouvait n'être, comme l'écrit D. Baggioni, que l'expression de normes d'usage, de normes objectives (ibid., p. 19), qui seront identifiées ultérieurement comme du FR. La question du rapport à la norme des FR constitue dès lors un enjeu essentiel.

\section{Le rapport à la norme : émergence de la notion de norme endogène, l'apport des études sociolinguistiques à la connaissance des français non hexagonaux}

\section{Les normes endogènes}

Penser la possibilité d'émergence de normes endogènes semble être la condition de base de la naissance de la notion de FR. Auguste Brun 6 , dans son étude du parler marseillais (1931), défend d'emblée l'intérêt du FR. Il considère que le provençal et le français qu'il nomme français commun (loc. cit.) s'enrichissent et s'influencent mutuellement. Ces interactions débouchent sur l'apparition entre le provençal et le français institutionnel d'une troisième variété qui constitue le FR (ibid., p. 6-9). C'est ainsi que, comme le notait D. Baggioni en 1995, du sentiment de l'existence d'une «spécificité du français régional» dépend le sentiment de "l'existence d'une norme plus ou moins assumée dans son autonomie» (1995, p. 74). Autrement dit, si le FR parvient, dans l'esprit des descripteurs, à incarner une norme à part entière, il ne sera pas assimilé à un ensemble de régionalismes plus ou moins stigmatisés et minorés. La notion de

6. Dans les années 1970, de manière similaire, Jean-Pierre Tennevin analysera les «provençalismes dans les devoirs d'élèves» (Tennevin, 1972), cité par P. Blanchet (2008, p. 66). 
régionalisme (Francard, Latin, 1995; Avanzi, 2017) est réductrice et ne convient pas aux variétés non hexagonales de français, car les situations de francophonie, hors hexagone, présentent une «palette de cas difficilement réductibles à une seule problématique» (Baggioni, 1993, p. 21). En effet, la notion de régionalisme suppose implicitement une hiérarchie verticale dans laquelle les variétés régionales constituent des satellites minorés du français central. Cette perspective est incompatible avec la revendication d'une francophonie linguistique multipolaire consubstantielle aux situations non hexagonales.

La francophonie non hexagonale semble ainsi avoir permis la «remise en question sociolinguistique de la langue standard » (Blanchet, Clerc, Rispail, 2014, p. 286). Les travaux des créolistes (Chaudenson, 1974, 1979a, 1979b et 1989; Hazaël-Massieux, 1978), les travaux sur les français d'Afrique (Manessy, 1978 et 1979; Manessy, Wald, 1984) ont permis de légitimer l'existence de «normes endogènes », et de théoriser la notion de FR (Baggioni, 1993, p. 24), tout comme les français «hors de France» (Valdman éd., 1979) ont constitué une source de «renouvellement de la réflexion sur le français régional» (Baggioni, 1993, p. 31).

\section{L'apport de la description des français non hexagonaux}

Les FR sont parlés par différentes communautés dont les locuteurs véhiculent des représentations sur la langue régionale, assumées, stigmatisées ou revendiquées, et sur lesquelles pèse la pression de la norme incarnée par le français central. C'est pourquoi il convient d'être attentif aux éléments extralinguistiques, et en particulier aux faits identitaires ou en lien avec la question des ethnicités (Baggioni, 1995, p.74), pour aborder les faits de langue. Les locuteurs du FR ne subissent pas la pression d'une norme explicite, résultant d'une vision uniformisée de la langue, mais celle d'une pluralité de normes, laquelle constitue la «principale assise de la francophonie» (Boulanger, 2001, p. 33) multipolaire dans laquelle se situe cette réflexion.

La dimension communicationnelle du FR est essentielle. Elle en détermine la dimension identitaire et situationnelle, si l'on admet que dans les situations de contacts de langues, de cultures, d'ethnicités, l'affirmation identitaire du locuteur peut primer sur la recherche d'une conformité de l'énoncé à la norme du français standard. Autrement dit, un locuteur identifiera une forme comme française ou créole moins en fonction de critères proprement linguistiques, qu'en fonction de ce qu'il sait de l'identité de son interlocuteur, de la situation de communication, les traits sociolinguistiques pouvant souvent l'emporter, au moins dans les variétés non standardisées, sur les propriétés linguistiques (Robillard, 1995, p. 27). De là provient la difficulté à définir la norme endogène dans la mesure où «la normalité qui la fonde n'est perçue que dans l'interaction même » (Manessy, 1993, p.17; Robillard, Beniamino, Bavoux, 1993, p. 25). 
Dans ces conditions, si l'on admet cette dimension du fonctionnement de la langue, quel est le statut de la communauté linguistique? La communauté linguistique francophone constitue-t-elle un ensemble homogène, et peut-on même parler de communauté?

\section{La communauté linguistique}

D'emblée, moins qu'une territorialisation des communautés linguistiques, il convient de postuler l'existence d'un réseau, plurilingue par nature, regroupant une large palette allant des locuteurs participant à l'élaboration et à la diffusion de la norme jusqu'aux locuteurs diglottes reconnaissant la norme mais la mettant en pratique de manière approximative, en passant par les adversaires de la norme du FR. Cette communauté linguistique doit être envisagée comme un espace social (Robillard, Beniamino, Bavoux, 1993, p. 36) où peut se développer un sentiment d'insécurité linguistique, qui conduit à évoquer une altérité minorée (Bertucci, 2006; 2016, p.173) à propos de cette situation de confrontation linguistique inégalitaire. On peut appliquer au $F R$ ce que Jean-Baptiste Marcellesi (Marcellesi, Bulot, Blanchet, 2003, p. 57) disait à propos de la minoration des langues régionales, à savoir que la notion de région a l'inconvénient de ne poser les problèmes qu'en termes géographiques en éludant les questions d'identités culturelles qui sont posées à travers elle. L'intérêt du terme minoré, en revanche, réside en ce qu'il fait appel au processus de minoration qui maintient dans une position subalterne, par l'intermédiaire de mécanismes économiques, sociaux, politiques, étatiques, des systèmes linguistiques virtuellement égaux au système officiel, la minoration ne résultant pas des différences linguistiques, mais de la dimension symbolique qui les entoure (ibid., p. 58).

La notion de région mérite d'être interrogée car elle a pris, dans le monde contemporain, une signification différente, qui lui confère des connotations qui ne sont plus celles que véhicule la notion de FR, dans son acception traditionnelle, et qu'on a vues jusque-là. On s'efforcera de montrer dans ce troisième temps que la notion de FR liée aux notions de normes et de développement endogènes est, en fait, très moderne dans un contexte postcolonial et mondialisé.

\section{L'opposition centre-périphérie. Intérêt de la notion de région pour l'analyse des politiques linguistiques-éducatives}

On voit apparaître le FR dans un triptyque français régional/français de Paris/ français standard. 


\section{Français régional/français de Paris/français standard}

On renvoie sur ce point aux travaux de Claude Poirier (1987), qui a construit la notion en opposant le FR au français de Paris, regroupant sous l'appellation français régional l'ensemble des variétés géographiques qui ne sont pas celles de Paris dans une perspective exclusivement topolectale, et à ceux de JeanClaude Corbeil, qui dans un article de 1984 intitulé «Le "français régional" en question » a élargi le concept en y intégrant la notion de communauté linguistique et celle d'espace géolinguistique.

Bernhard Pöll met en débat le mot région et se demande s'il est bien approprié à des territoires étendus comme le Québec ou la Belgique (Pöll, 2001, p. 31). Ce débat est également présent chez C. Poirier (1987, p.154). B. Pöll préfère les termes de variété nationale ou de français de Belgique ou du Québec, ou encore de français territorial (Pöll, 1998, p. 170). Ces chercheurs attribuent au mot région un sémantisme restreint (Pöll, 1998, p. 31-32 ; Poirier, 1987, p.152). Si la Provence est bien une région, cela n'est pas le cas de la Suisse ou du Cameroun à leurs yeux (Pöll, 1998).

Or, selon nous, ce point de vue est discutable, et ce particulièrement dans le contexte mondialisé, dans lequel on souhaite inscrire cette réflexion sur les FR. Le mot région est polysémique et l'espace que le terme désigne revêt dans certains contextes une surface beaucoup plus étendue.

\section{L'échelon régional: un niveau de réflexion pour les politiques linguistiques-éducatives}

On souhaiterait ouvrir la réflexion à ce stade en interrogeant non plus la notion de français, mais celle de région, qu'on pourra également dénommer « échelon régional» ou encore «intégration régionale» (Bertucci, 2016, p. 179).

La dimension économique de la question ne sera pas abordée ici bien que la notion d'intégration régionale soit empruntée à cette discipline. En effet, si l'intégration régionale constitue d'abord et principalement un « rapprochement commercial, économique, institutionnel entre États» (Kauffmann, Yvars éd., 2004, p.7), elle est aussi pertinente au niveau linguistique et culturel. La région7 occupe une place intermédiaire entre la structure de base qu'est la commune et le niveau central ou fédéral de l'État (D’Angelo, Vespérini, 2000, p. 13). Elle se situe entre le local et le national et permet de focaliser les relations sur une zone. Sans approfondir la dimension institutionnelle de la définition, on

7. Ce que recouvre le mot région est problématique et on observe une grande amplitude de dénominations : «province, land, communauté» (D’Angelo, Vespérini, 2000, p.7). Elle peut renvoyer également à une entité résultant d'un découpage administratif, comme la région Centre en France, et être indépendante de l’héritage historique et de la réalité linguistique et culturelle. 
s’intéressera à la réalité linguistique de cette entité, à ce qui inspire « un sentiment d'appartenance à un territoire et/ou à une communauté distinctive», linguistique en particulier (ibid., p. 9). La région peut constituer un « ensemble territorial » ancré dans une appartenance linguistique (ibid., p. 15) ou se manifester comme une communauté linguistique et culturelle «sans ancrage territorial ${ }^{8}($ loc. cit.). La place intermédiaire de la région entre les échelons locaux et nationaux lui confère 9 à la fois une plus grande proximité avec le pouvoir de décision national ${ }^{10}$ que le niveau local, et une aptitude à exprimer pleinement une identité régionale, notamment en termes d'appartenance linguistique et culturelle, laquelle est peu visible, voire minorée, au niveau national. Enfin, la réflexion sur la notion de région conduit à reformuler la question et la place de l'État-nation dans le contexte de la mondialisation et à dépasser les limites du territoire national (Hugon éd., 2002, p. 10) pour se situer à un niveau transfrontalier. Ceci suppose de reconnaître qu'il existe des zones transfrontalières ${ }^{11}$ où vit une population dotée de caractéristiques linguistiques identiques et d'accepter de fonder les politiques linguistiques-éducatives sur l'histoire locale et la culture, et donc de les contextualiser. On trouve de nombreux exemples de cette situation en Afrique, mais aussi en Europe. Pascal Kauffmann et Bernard Yvars citent la région de Basiliensi12 (2004, p.63). Ce type de situation remet en cause la notion de frontière, conduit à prendre en compte les minorités et à privilégier des sous-ensembles régionaux eux-mêmes en relation avec le contexte mondial (Ténier, 2009, p.126; Eloy éd., 2018, p.12). Réfléchir aux politiques linguistiques-éducatives à un niveau régional suppose aussi de mettre en place un processus multidimensionnel conduisant à une intrication entre les espaces nationaux, à une convergence de projets et à une harmonisation des politiques, ce qui n'est pas sans conséquence sur la souveraineté des États. L'intégration régionale est à l'origine de la constitution d'un nouvel espace (Ténier, 2003, p.10), qui modifie le processus de la mondialisation et le nuance, dans la mesure où la régionalisation est complémentaire de la globalisation tout en la contredisant (ibid., p. 9).

Dans le domaine des politiques linguistiques-éducatives, les normes endogènes, et tout ce qui s’inscrit dans le cadre de la réflexion sur les problèmes posés par «l'acclimatation du français » (Blanchet, Clerc, Rispail, 2014,

8. La minorité suédophone a reçu dès 1920 l'autonomie culturelle en Finlande (D'Angelo, Vespérini, 2000, p.15).

9. À condition que le découpage régional soit fait selon des critères en référence à des appartenances historiques, qu'elles soient linguistiques ou culturelles.

10. L'instance de décision restant assez près de la population (D’Angelo, Vespérini, 2000, p. 52).

11. Sur la notion de zones transfrontalières, voir Kauffmann, Yvars éd., 2004, p. 70 et suiv.

12. Laquelle a été créée en 1963 autour de la «trinationale» (Suisse, France, Allemagne) ville de Bâle, qui est au cœur d'une région transfrontalière s'étendant sur le Jura suisse, la Forêt-Noire allemande et les Vosges françaises et où est parlé un dialecte allemand (Kauffmann, Yvars éd., 2004, p.63). 
p. 286) ${ }^{13}$, pourraient constituer la base d'un enseignement du français contextualisé dans l'espace de la région, dans le cadre d'un aménagement linguistique fondé sur un modèle pluricentrique (Pöll, 2005, p. 252). Il conviendrait également de trouver une démarche didactique adaptée qui prenne en considération l'identité culturelle et linguistique des élèves, sans les y enfermer, et qui tienne compte du fait que la perception qu'aura l'élève de la langue à apprendre, ici le français de scolarisation, va fortement en conditionner l'apprentissage. Ceci suppose une réflexion d'une part sur le statut du français de scolarisation et sur les représentations que les enseignants et les élèves se sont forgées de celui-ci, et d'autre part sur la gestion de l'insécurité linguistique et les processus d'inclusion/exclusion qu'elle suscite dans le cadre de la classe. La lexicographie francophone ${ }^{14}$ peut s'avérer une ressource particulièrement pertinente dans cette perspective didactique.

\section{L’apport de la lexicographie francophone à la didactique du français : les enjeux didactiques de la variation}

La lexicographie francophone pose le problème de la frontière et du contact entre variétés de français, et notamment la question des phénomènes d'appropriation et de créativité, en particulier des variétés africaines, pour reprendre le sous-titre de l'ouvrage de Suzanne Lafage, Le lexique français de Côte d'Ivoire : appropriation et créativité (2003). On peut y voir un mouvement d'appropriation d'un terme et de création d'un sens nouveau. Ainsi, ration signifie «la somme d'argent destinée à l'achat de la nourriture de base journalière de la famille» (Lafage, 2003, p. 698), piétiner ou gratter: «aller à pied» (ibid., p. 450 et 652), individu : « imbécile ou personne peu recommandable» (ibid., p. 488), être riénard : «être sans le sou» (ibid., p. 708).

La ressource didactique que constituent les FR est néanmoins peu exploitée dans le cadre scolaire. Ainsi les manuels scolaires utilisés à La Réunion ne mentionnent pas les particularités lexicales régionales (Carayol, 1984, p. 6). On peut l'attribuer au fait que le français régional réunionnais est, dans une certaine mesure, une langue occultée, sans légitimité (Beniamino, 1996, p.14) en dehors de sa dimension topolectale et donc non légitime dans le cadre scolaire.

Ainsi, des termes du lexique créole, d'un usage courant en FR, sont écartés de l'usage scolaire, alors qu'ils renvoient à un quotidien qu'il est difficile, voire impossible, de désigner autrement (Carayol, 1984, p. 5-6). De nombreux

13. Cette notion a été forgée par P. Blanchet, S. Clerc et $M$. Rispail dans le cadre d'une réflexion sur les questions sociolinguistiques et didactiques au Maghreb.

14. Compte tenu de l'ampleur de ce sujet, on ne peut aborder l'ensemble des champs des sciences du langage que recouvre la question de l'étude de la langue en contexte scolaire. Pour les questions d'orthographe, on renvoie notamment à l'article de P. Blanchet (1994). 
termes ayant un signifiant identique en créole et en français, mais offrant dans les deux langues une opposition sémantique plus ou moins forte, coexistent. Ces termes ne sont pas identifiés par les élèves et très souvent ne le sont pas par les maîtres, ce qui conduit à pérenniser et à consolider des confusions sémantiques qui perturbent l'acquisition et la maîtrise des deux langues. Michel Carayol conclut à la nécessité d'intégrer dans la pratique scolaire du français à La Réunion les particularités lexicales renvoyant à la réalité quotidienne. L'homonymie, la synonymie, essentielles dans les FR, ne reçoivent pas forcément le traitement didactique requis à l'école, faute de politiques linguistiques-éducatives fondées sur un enseignement du français contextualisé et en phase avec la situation linguistique locale. Ce hiatus peut expliquer pour partie les difficultés massives des élèves dans l'apprentissage du français. On mesure ici l'importance, pour les enseignants, de l'existence d'outils comme ceux que constituent les «dictionnaires globaux»15, qui autorisent la mise en place d'un "processus d'intégration des "mots de la francophonie" » (Bavoux, 2008, p. 345), notamment dans des situations scolaires où seraient en présence des élèves venus de différents points de l'espace francophone.

On reviendra pour conclure sur les conséquences de cette minorisation des FR sur les locuteurs et, dans le cadre de l'école, sur les élèves.

L'indifférence de l'école à l'égard de la langue des élèves peut susciter chez ces derniers un sentiment d'infériorité, voire d'anomie, et renforcer de fait le sentiment d'appartenir à un groupe minoritaire, peu ou pas assez reconnu. Or, reconnaître l'existence des FR ne signifie pas pour autant marginaliser les individus, mais peut constituer au contraire une marque de reconnaissance, un signe de légitimation pour une meilleure intégration. L’identité commune peut s'édifier sur des composants variés et ne suppose pas forcément une totale uniformité. En effet, la diversité culturelle constitue un trait de la vie nationale française. Enfin, une conception essentialiste de la langue domine souvent l'approche du français (Klinkenberg, 2001, p.60).

On observera au bout du compte que les représentations construites autour du français peuvent être interprétées comme particulièrement significatives de cette relation inégalitaire de hiérarchie entre les différentes variétés de français en présence et supposent d'être modifiées pour rendre possible une évolution vers la légitimation des normes endogènes plurielles (Marcellesi, Romian, Treignier, 1985), évolution dans le prolongement de laquelle pourrait se mettre en place « une didactique de la pluralité linguistique» (Blanchet, Clerc, Rispail, 2014, p. 296).

Enfin, on soulignera que la problématique n'est pas exclusivement sociolinguistique. Elle est également économique et politique à un double niveau

15. On donnera l'exemple du Dictionnaire universel francophone (DUF), Hachette-Edicef (1997), cité par C. Bavoux (2008, p. 345). Cet ouvrage met en regard en un unique volume la diversité des variétés endogènes de français dans l'espace francophone. 
macro- et micro-sociolinguistique/économique (Robillard, 1989, p.63) dans le cadre d'une relation entre langues et développement. La condition est d'admettre le recours à des modèles de développement endogènes dans un contexte multipolaire et globalisé (Ki-Zerbo, 2003, p.167) et de conduire une réflexion sur l'aménagement linguistique (Moussirou-Mouyama, 2009 [2010], p.37), et notamment sur la place des variétés endogènes de français, dans la perspective d'une francophonie conçue comme un «projet humaniste et citoyen » (ibid., p.40), pour permettre d'aller vers un « développement durable» (ibid., p. 36). Les langues apparaissent comme le socle incontournable des politiques de développement (Chaudenson, Robillard, 1989), lesquelles doivent être fondées sur les caractéristiques linguistiques et culturelles des populations concernées. Ces différents éléments conduisent à s’interroger sur la notion de «frontière » et de «lien langue-État» dans une perspective tant linguistique que géopolitique, laquelle amène aussi à questionner la notion de communauté francophone comme "communauté virtuelle» sans "continuum physique» (MoussirouMouyama, 2009 [2010], p. 41) et comme communauté linguistique et culturelle « sans ancrage territorial » (D’Angelo, Vespérini, 2000, p. 15), ou encore, dans le cadre de l'intégration européenne, à problématiser la notion de « cosmopolitanisme », conçue par Chris Rumford (2008) comme un espace cosmopolite fondé sur une politique d'espaces publics et discursifs. Vue sous cet angle, la prise en compte des FR dans les politiques linguistiques-éducatives acquiert une pertinence certaine, car elle permet d'enter les stratégies de développement sur des réalités langagières effectives et sur les pratiques avérées des locuteurs.

\section{Références}

Avanzı Mathieu, 2017, Atlas du français de nos régions, Malakoff, Armand Colin.

BAGGIONI Daniel, 1995, «Variante géographique du français? Remarques sur les présupposés théoriques et la grille de classement de C. Poirier », dans Le régionalisme lexical, M. Francard et D. Latin éd., Louvain-la-Neuve, Bruxelles, Duculot, De Boeck Université, p. 67-77.

- 1993, "Historique du concept de français régional», Travaux \& documents - Université de La Réunion, Faculté des Lettres et sciences humaines, nº 3, p. 17-38.

Bavoux Claudine, 2008, "Pour ne pas conclure», dans Le français des dictionnaires: l'autre versant de la lexicographie française, C. Bavoux éd., Bruxelles, De Boeck, Duculot, p. 337-351.

BeniAmino Michel, 1996, Le français de La Réunion : inventaire des particularités lexicales, Vanves, Edicef.

BÉRARD Léon, 1995, "20 juin 1923. Instructions sur les nouveaux programmes des écoles primaires», dans L'enseignement du français à l'école primaire, t.II : 18801939, A. Chervel éd., Paris, INRP, p.313-331, https://www.persee.fr/doc/inrp_00oo0000_1995_ant_5_2_2072 (consulté le 1er avril 2019). 
Bertuccı Marie-Madeleine, 2016, «Modalités d'une altérité minorée. Pertinence de la notion de région pour les politiques linguistiques-éducatives», dans Les français régionaux dans l'espace francophone, M.-M. Bertucci éd., Francfort, Peter Lang, p. $173-185$.

- 2006, Plurilinguisme et altérité: français, école, politiques linguistiques-éducatives, note de synthèse pour l'habilitation à diriger des recherches, Université de Tours, non publiée.

BLANCHET Philippe, 2016, "De l'observation des variétés locales et régionales du français à une didactique de la pluralité des pratiques du français en contexte plurilingue : un chemin semé d'embûches », dans Les français régionaux dans l'espace francophone, M.-M. Bertucci éd., Francfort, Peter Lang, p. 17-30.

- 2008, "Vingt-cinq ans de construction du "français régional de Provence" (19822007)", dans Le français des dictionnaires : l'autre versant de la lexicographie française, C. Bavoux éd., Bruxelles, De Boeck, Duculot, p. 63-75.

- 1994, "Variations orthographiques et variétés régionales du français : des pistes didactiques », Le français aujourd'hui, no 107, p. 112-118.

Blanchet Philippe, Clerc Stéphanie, RISPAIL Marielle, 2014, «Réduire l'insécurité linguistique des élèves par une transposition didactique de la pluralité sociolinguistique : pour de nouvelles perspectives sociodidactiques avec l'exemple du Maghreb », dans Insécurité linguistique en éducation : approche sociologique comparée des élèves issus du Maghreb, B. Garnier éd., Paris, Didier érudition, Klincksieck, p. 283-302.

BOULANGER Jean-Claude, 2001, "La francophonie : une norme, des normes, un dictionnaire, des dictionnaires?», dans Variations et dynamismes du français : une approche polynomique de l'espace francophone, F. Laroussi et S. Babault éd., Paris, L'Harmattan, p. 31-49.

BRUn Auguste, 1931, Le français de Marseille : étude de parler régional, Marseille, Institut historique de Provence.

Carayol Michel, 1984, Particularités lexicales du français réunionnais : propositions pédagogiques, Paris, Nathan.

Chaudenson Robert, 1989, Créoles et enseignement du français, Paris, L'Harmattan.

- 1979a, "Le français dans les îles de l'océan Indien (Mascareignes et Seychelles)», dans Le français hors de France, A. Valdman éd., Paris, Honoré Champion, p. 543-617.

- 1979b, Les créoles français, Paris, Nathan.

- 1974, Le lexique du parler créole de La Réunion, Paris, Honoré Champion.

Chaudenson Robert, Robillard Didier de, 1989, Langues, économie et développement, Aix-en-Provence, Paris, Institut d'études créoles et francophones, Didier érudition, tome I.

CORBEIL Jean-Claude, 1984, "Le "français régional" en question », dans Langues et cultures : mélanges offerts à Willy Bal, vol. II : Contacts de langues et de cultures, C. Faïk-Nzuji M. éd., Louvain-La-Neuve, Cabay, Institut de linguistique de Louvain, p. 31-44.

D’Angelo Mario, Vespérinı Paul, 2000, Politiques culturelles en Europe : régions et décentralisation culturelle, Strasbourg, Éditions du Conseil de l’Europe. 
DAUZAT Albert, 1933, "La diffusion du français en France et le français régional», Le français moderne, $\mathrm{n}^{0} 1, \mathrm{p} .133-143$.

- 1906, Essai de méthodologie linguistique dans le domaine des langues et patois romans, Paris, Honoré Champion.

ELoY Jean-Michel éd., 2018, De France et d'au-delà : les langues régionales transfrontalières, Paris, L'Harmattan.

FRANCARD Michel, 2010, «Variation diatopique et norme endogène. Français et langues régionales en Belgique francophone », Langue française, nº 167, p.113-126.

- 1993, «Entre Romania et Germania : la Belgique francophone», dans Le français dans l'espace francophone: description linguistique et sociolinguistique de la francophonie, D. de Robillard et M. Beniamino éd., Paris, Honoré Champion, t. I, p.317-336.

Francard Michel, LATIN Danièle, 1995, Le régionalisme lexical, Louvain-la-Neuve, Bruxelles, Duculot, De Boeck Université.

GADET Françoise, 2007 [2003], La variation sociale en français, Paris, Ophrys.

HAZAËL-MASSIEUX Guy, 1978, «Approche socio-linguistique de la situation de diglossie français-créole en Guadeloupe », Langue française, n³7, p. 106-118.

Huck Dominique, KaHn René, 2009, Langues régionales, cultures et développement : études de cas en Alsace, Bretagne et Provence, Paris, L'Harmattan.

Hugon Philippe éd., 2002, Les économies en développement à l'heure de la régionalisation, Paris, Karthala.

KAUfFMAnn Pascal, Yvars Bernard éd., 2004, Intégration européenne et régionalisme dans les pays en développement, Paris, L'Harmattan.

KI-Zerbo Joseph, 2003, À quand l'Afrique? Entretien avec René Holenstein, La Tour d'Aigues, Éditions de l'Aube.

KLINKENBERG Jean-Marie, 2001, La langue et le citoyen : pour une autre politique de la langue française, Paris, PUF.

LAFAGE Suzanne, 2003, Le lexique français de Côte d'Ivoire : appropriation et créativité, Paris, Nice, Institut de linguistique française, UMR Bases, Corpus, Langage.

MANESSY Gabriel, 1993, "Normes endogènes et français de référence », dans Inventaire des usages de la francophonie : nomenclatures et méthodologies, D. Latin, A. Queffelec et J. Tabi Manga éd., Montrouge, Londres, Rome, Montréal, J. Libbey-Eurotext, AUPELF, p. 15-23.

- 1979, "Le français en Afrique noire, faits et hypothèses », dans Le français hors de France, A. Valdman éd., Paris, Honoré Champion, p. 333-362.

- 1978, "Le français d'Afrique noire, français créole ou créole français ", Langue française, n³ 37, p. 91-105.

MANESSY Gabriel, WALD Paul, 1984, Le français en Afrique noire, tel qu'on le parle, tel qu'on le dit, Paris, L'Harmattan.

MarCellesI Jean-Baptiste, Bulot Thierry, Blanchet Philippe, 2003, Sociolinguistique: épistémologie, langues régionales, polynomie, Paris, L'Harmattan.

MARCELlesı Christiane, Romian Hélène, TREIGNIER Jacques, 1985, "Quelques concepts et notions opératoires pour une pédagogie de la variation langagière», Repères, n०67, p. 23-31.

Ministère de l'éducation nationale, 1938, Enseignement du premier degré: instructions 
du 20 septembre 1938 relatives à l'application des arrêtés du 23 mars 1938 et du 11 juillet 1938, Paris, Vuibert.

MoussiRou-Mouyama Auguste, 2009 [2010], «Hétérogénéité de l'Afrique et réussite sociale : que peut-on faire avec la linguistique pour lutter contre l'échec scolaire en Afrique francophone?», Cahiers de linguistique, n³5/2, p. 33-47.

MüLler Bodo, 1985, Le français d'aujourd'hui, A. Elsass trad., Paris, Klincksieck.

PoIRIER Claude, 1995, «Les variantes topolectales du lexique français, Propositions de classement à partir d'exemples québécois», dans Le régionalisme lexical, M. Francard et D. Latin éd., Louvain-la-Neuve, Bruxelles, Duculot, De Boeck Université, p.13-56.

- 1987, "Le français "régional" : méthodologies et terminologies», dans Français du Canada, français de France, H.-J. Niederehe et L. Wolf éd., Tübingen, Niemeyer, p. $139-176$.

PöLL Bernhard, 2005, Le français langue pluricentrique? Études sur la variation diatopique d'une langue standard, Francfort, Peter Lang.

- 2001, Francophonies périphériques : histoire, statut et profil des principales variétés du français hors de France, C. Ollivier trad., Paris, L'Harmattan.

- 1998, "Le français ou les français ? La difficile naissance de la pluricentricité », Lengas. Revue de sociolinguistique, $\mathrm{n}^{\circ} 43$, p. 163-182.

RÉmOND René, 1974, Introduction à l'histoire de notre temps. 3, Le xxe siècle de 1914 à nos jours, Paris, Seuil.

Riegel Martin, Pellat Jean-Christophe, Rıoul René, 2016, Grammaire méthodique du français, Paris, PUF.

RoBILLARD Didier de, 1995, «Lexicographie variationniste, linguistique, sociolinguistique : antagonisme ou complémentarité ? Prédictibilité aléatoire ou chaos?», dans Le régionalisme lexical, M. Francard et D. Latin éd., Louvain-la-Neuve, Bruxelles, Duculot, De Boeck Université, p.185-202.

- 1989, "Vers une approche globale des rapports entre langue et économie», dans R. Chaudenson et D. de Robillard, Langues, économie et développement, Aix-enProvence, Paris, Institut d'études créoles et francophones, Didier érudition, t.l, p. 39-65.

Robillard Didier de, Beniamino Michel, Bavoux Claudine, 1993, "Le français dans l'espace francophone : problématique», dans Le français dans l'espace francophone : description linguistique et sociolinguistique de la francophonie, D. de Robillard et M. Beniamino éd., Paris, Honoré Champion, t. I, p.17-48.

RUMFORD Chris, 2008, Cosmopolitan Spaces: Europe, Globalization, Theory, Londres, Routledge.

TAVERDET Gérard, 1977, «Le français régional dans la côte bourguignonne », Travaux de linguistique et de littérature, vol.XV, nº 1, p. 35-42.

TAVERDET Gérard, StRAKA Georges, 1977, Les français régionaux, Paris, Klincksieck.

TÉNIER Jacques, 2009, «Les enseignements de l'intégration régionale pour la construction d'un avenir commun aux Africains et aux Européens », dans L'Union européenne et la montée du régionalisme : exemplarité et partenariats, C. Franck, J.-C. Defraigne et V. de Moriamé éd., Louvain-la-Neuve, Bruylant-Academia, p.125-141.

- 2003, Intégrations régionales et mondialisation : complémentarité ou contradic- 
tion, Paris, La Documentation française.

TENNEVIN Jean-Pierre, 1972, "Les provençalismes dans les devoirs d'élèves », Le français aujourd'hui, nº 19, p. 50-55.

TuAILlon Gaston, 1983, Les régionalismes parlés à Vourey, village dauphinois, Paris, Klincksieck.

- 1977, «Réflexions sur le français régional », Travaux de linguistique et de littérature, vol.XV, $\mathrm{n}^{0} 1, \mathrm{p} .7-29$.

VALDMAN Albert éd., 1979, Le français hors de France, Paris, Honoré Champion.

WALter Henriette, 1984, "Patois ou français régional?", Le français moderne, nº 52 , p. 183-190.

ZAY Jean, 1938, Enseignement du premier degré. Instructions du 20 septembre 1938 relatives à l'application des arrêtés du 23 mars 1938 et du 11 juillet 1938, Paris, Vuibert, https://www.samuelhuet.com/paid/41-textes-officiels/946 (consulté le 25 avril 2019).

\section{Résumé / Abstract / Compendio}

\section{Vers une conceptualisation de la notion de «français régional» : de la dialec- tologie à la sociolinguistique}

L'article se développe en trois temps. Il voit d'abord comment la notion de français régional a évolué des premiers travaux d'Albert Dauzat jusqu'aux analyses les plus récentes. Il analyse ensuite l'émergence de la notion de norme endogène, les apports des études sociolinguistiques consacrées aux français non hexagonaux, notamment ceux de la lexicographie francophone, à l'étoffement de la notion de français régional. Il s'interroge enfin sur la modernité de la notion de région dans le contexte mondialisé contemporain et sur la revitalisation qu'elle confère à la notion de français régional.

Mots-clés: espace francophone, dialectologie, sociolinguistique, français régional, norme endogène, région

\section{Towards a conceptualization of the notion of regional French: from dialectology to sociolinguistics}

The article develops in three stages. It first shows how the notion of regional French has evolved from Albert Dauzat's first works to the most recent analyses. It then analyzes the emergence of the notion of endogenous norm, the contributions of sociolinguistic studies devoted to non-hexagonal French, especially those of French-speaking lexicography, and the expansion of the notion of regional French. Finally, it questions the modernity of the notion of region in a contemporary globalized context and the revitalization it confers on the notion of regional French.

Keywords: French-speaking world, dialectology, sociolinguistics, regional varieties of French, diatopical norm, region

\section{Hacia una conceptualización de la noción de «francés regional»: desde la dialectología a la sociolingüística}

El artículo se desarrola en tres etapas. Primero se determina cómo la noción de francés regional se desarrolló desde los primeros estudios de Albert Dauzat hasta los más 
recientes. Trata despues de la emergencia de la noción de norma endógena, de los aportes de los estudios sociolingüísticos consagrados a los franceses hablados fuera del territorio, y notamente por los de la lexicografía francófona, al enriquecimientio de la noción de francés regional. Para terminar, se interroga la modernidad de la noción de región dentro del contexto mundializado contemporaneo y su impacto sobre la revitalización que confiere a la noción de francés regional.

Keywords: espacio francófono, dialectología, sociolingüística, francés regional, norma endogena, región 